\title{
Catheter directed thrombolytic therapy and aspiration thrombectomy in intermediate pulmonary embolism with long term results
}

\author{
Zoltan Ruzsa ${ }^{1,2}$, Zoltan Vámosi², Balázs Berta ${ }^{3}$, Balázs Nemes ${ }^{1}$, Károly Tóth ${ }^{2}$, \\ Nándor Kovács ${ }^{2}$, Endre Zima ${ }^{1}$, Dávid Becker ${ }^{1}$, Béla Merkely ${ }^{1}$ \\ ${ }^{1}$ Semmelweis University of Budapest, Heart and Vascular Center, Budapest, Hungary \\ ${ }^{2}$ Bács-Kiskun County Hospital, Cardiology Division, Invasive Cardiology, Kecskemét, Hungary \\ ${ }^{3}$ Department of Cardiology, Isala Hospital, Zwolle, The Netherlands
}

\begin{abstract}
Background: Catheter directed thrombolysis (CDT) and thrombectomy represent well established techniques for the treatment of intermediate pulmonary embolism (IPE). The long-term effect of catheter directed thrombolysis of IPE is unknown.

Methods: Clinical, interventional and echocardiographic data from 80 consecutive patients with IPE who were treated with CDT were evaluated. Primary end-points were technical success and major adverse events. Secondary end-points were cardiovascular mortality, all-cause mortality, clinical success, rate of bleeding complications, improvement in pulmonary pressure and echocardiography parameters. CDT completed with alteplase (10 $\mathrm{mg}$ bolus and $1 \mathrm{mg} / \mathrm{h}$ maintenance dose) through a pig-tail catheter for $24 \mathrm{~h}$. After $24 \mathrm{~h}$, control pulmonary angiography was performed.

Results: In total, 80 patients with a mean age of $59.0 \pm 16.8$ years were treated. CDT was successful after the first post-operative day in 72 (90\%) patients, but thrombus aspiration and fragmentation was performed due to failed thrombolysis in $8(10 \%)$ patients. Final technical and clinical success was reached in 79 (98.8\%) and 77 (96.3\%) patients, respectively. The mean CDT time in IPE was $27.8 \pm$ \pm 9.6 h. Invasive pulmonary pressure dropped from $57.5 \pm 16.7$ to $38.9 \pm 13.5(p<0.001)$. A caval filter was implanted in $4(5 \%)$ patients. The 1-year major adverse events and cardiovascular mortality rate was $4.0 \%$ and 1.4\%, respectively. Access site complications (6 major and 6 minor) were encountered in $12(16.2 \%)$ patients.

Conclusions: Catheter directed thrombolysis in submassive pulmonary embolism had excellent results. However, additional mechanical thrombectomy was necessary in some patients to achieve good clinical outcomes. (Cardiol J 2020; 27, 4: 368-375)
\end{abstract}

Key words: pulmonary embolism, local thrombolysis

\section{Introduction}

Venous thromboembolic disease is an underdiagnosed disease spectrum with a high mortality and morbidity rate. Recent advances in interventional cardiology, pharmacotherapeutics and modern surgical management in tertiary cardiac care centers have tremendously improved the present treatment of pulmonary embolism (PE). PE is commonly classified as high-risk, intermediaterisk, and low-risk to help determine the required treatment [1]. Despite recent advances in mechanical thrombectomy (MT) and catheter directed thrombolysis (CDT), the primary treatment is systemic thrombolysis (ST) in intermediate- and high-risk $\mathrm{PE}$ [1]. ST is associated with a high rate of

Address for correspondence: Zoltán Ruzsa, MD, PhD, Associate Professor, Semmelweis University, Heart and Vascular Center, Budapest, 1122, Városmajor Street 68, Hungary, tel: 0036203338490, e-mail: zruzsa25@gmail.com

Received: 2.12.2019

Accepted: 2.02.2020 


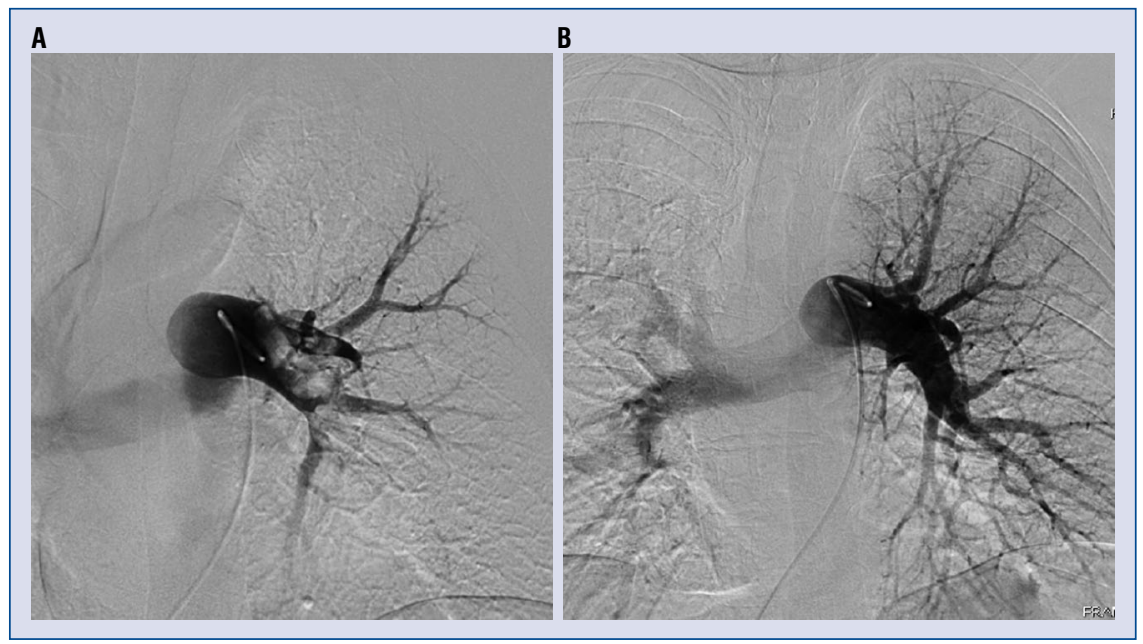

Figure 1. Angiography and catheter directed thrombolysis of a 63-year-old woman presenting with acute dyspnea and referred for pulmonary angiography; A. Selective angiography from right femoral vein access shows nearly complete left main pulmonary artery occlusion and elevated pulmonary pressures. Selective thrombolysis with $10 \mathrm{mg}$ tissue plasminogen activator, followed by $1 \mathrm{mg} / \mathrm{h}$-maintained infusion was initiated; B. Control angiography shows complete resolution of the thrombi and decreased pulmonary pressure.

recanalization in intermediate PE (IPE), but bleeding complications are very frequent and hazardous [2]. Despite recent advances in IPE, MT and CDT have a IIb indication in the recent European and American College of Cardiology guidelines [1, 2]. When thrombolysis is contraindicated or has failed, urgent surgical embolectomy or catheter embolectomy may be lifesaving procedures in high-risk $\mathrm{PE}$. The aim of the study was to investigate the safety and efficacy of CDT and MT in IPE and to investigate the long-term effects on functional status and pulmonary pressure.

\section{Methods}

Clinical, interventional and echocardiographic data from 80 consecutive patients with IPE who were treated with CDT were evaluated. Diagnosis was made by computed tomography or pulmonary angiography and transthoracic ultrasound.

Inclusion criteria were: intermediate pulmonary embolism (defined as normotensive patients with $\mathrm{PE}$ and evidence of right ventricular dysfunction) and patients who underwrote the patient form.

Exclusion criteria were: contraindications for the local administration of thrombolytic therapy (stroke or transient ischemic attack, head trauma, or other active intracranial or intraspinal disease within 12 months; major surgery within 7 days; recent active bleeding from a major organ); and massive PE (defined as syncope, systemic arterial hypotension, cardiogenic shock, or resuscitated cardiac arrest).
Primary end-points were: technical success and major adverse events.

Secondary end-points were: all-cause mortality, cardiovascular mortality, clinical success, rate of bleeding complications, improvement in pulmonary pressure, blood gas analysis, and echocardiography parameters.

\section{Procedure description}

The access site for CDT was the contralateral femoral vein. The common femoral vein was punctured in a regular fashion, but in vascular cases, vascular ultrasound guidance was used when the puncture was difficult. Pulmonary angiography and invasive pulmonary pressure measurement were performed before CDT (Fig. 1A). CDT was done with alteplase $(10 \mathrm{mg}$ bolus and $1 \mathrm{mg} / \mathrm{h}$ maintenance dose) through a pig-tail catheter for $24 \mathrm{~h}$. After $24 \mathrm{~h}$, control pulmonary angiography and invasive pressure measurement was done (Fig. 1B). During the procedure, intravenous unfractionated heparin was continued at intermediate intensity with a goal activated partial thromboplastin time of 40-60 s. MT was performed when the thrombus burden was flow limiting or the systolic pulmonary pressure had not decreased by $25 \%$ or to the normal level (Fig. 2A). Thrombus fragmentation with the pigtail catheter was done in cases of extreme flow limiting thrombus. Thrombus aspiration was done with a $7 \mathrm{~F}$ guiding catheter and long $7 \mathrm{~F}$ sheath using a 50-mL syringe for manual aspiration (Fig. 2B). After removal of the catheters, the access site was manually compressed for a minimum of 


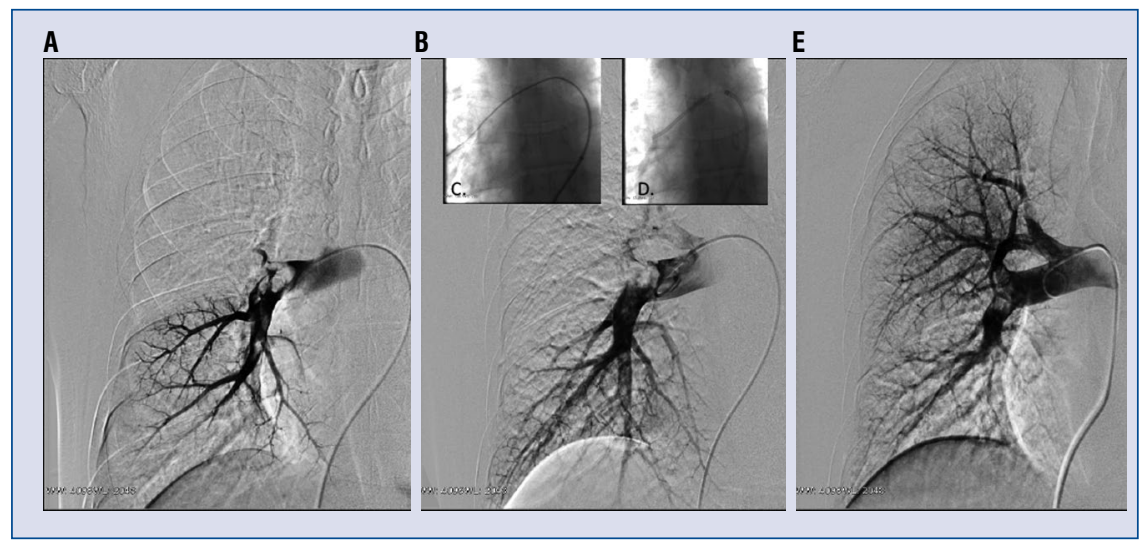

Figure 2. Catheter directed thrombolysis and mechanical thrombectomy of a 68-year-old man presenting with acute dyspnea; A. Selective angiography from right femoral vein access shows nearly complete right main pulmonary artery occlusion and elevated systolic pulmonary pressure $(70 \mathrm{mmHg})$. Selective thrombolysis with $10 \mathrm{mg}$ tissue plasminogen activator, followed by $1 \mathrm{mg} / \mathrm{h}$-maintained infusion was initiated; B. Control angiography shows incomplete thrombus resolution and hemodynamic result (systolic pulmonary pressure: $70 \mathrm{mmHg}$ ), therefore a $7 \mathrm{~F} 90 \mathrm{~cm}$ sheath was advanced in the right main pulmonary artery over a long $360 \mathrm{~J}$ tip guidewire (C) and mechanical thrombectomy was done with a $100 \mathrm{~cm}$ Judkins right guiding catheter (D); E. Control angiography shows complete resolution of the thrombi and decreased systolic pulmonary pressure $(40 \mathrm{mmHg})$.

5 min. Insertion of inferior vena cava filters was discouraged unless the patient developed a contraindication to therapeutic-dose systemic anticoagulation or if the patient suffered recurrent PE despite therapeutic levels of anticoagulation. Postoperatively, patients were treated with systemic anticoagulation and compression hose, and follow-up was completed at specified intervals.

Pulmonary angiography with digital subtraction was done on the next postoperative day in all patients. Thrombus burden was categorized as: occlusive or large (75-100\% of the lumen) (Grade 3), medium (50-75\% of the lumen) (Grade 2), small $(0-50 \%$ of the lumen) (Grade 1$)$ or no visible thrombus (Grade 0).

Twelve-lead electrocardiography was done in all patients at admission and before discharge.

Transthoracic ultrasound was performed before and after thrombolytic therapy. Routine right heart measurements were performed to investigate right ventricular function.

\section{Post procedural follow-up}

Access arteries were investigated physically after the procedure. All patients underwent detailed clinical follow-up examinations at two and 12 months after the procedure.

\section{Definitions}

Pulmonary hypertension was quantified by systolic pulmonary artery pressure as normal
( $<40 \mathrm{mmHg}$ ), mild hypertension ("40-60" > $>90 \mathrm{mmHg}$ ), moderate hypertension ("60-90 " > $90 \mathrm{mmHg}$ ) and severe hypertension (>90 mmHg) [3].

Major adverse events were assessed as the composite of cardiovascular death, myocardial infarction, stroke, and repeated revascularization of the target vessel by surgical thrombectomy at 2 months and 12 months.

Bleeding events were classified by the Global Utilization of Streptokinase and Tissue Plasminogen Activator for Occluded Coronary Arteries (GUSTO) bleeding criteria [3]; major bleeding was defined as either GUSTO moderate or GUSTO severe/life-threatening bleeding events.

A major vascular access complication was defined as a diminished or lost arterial pulse or the presence of any pseudoaneurysm or arteriovenous fistula during clinical follow-up. A minor complication was defined as a hematoma requiring no further treatment that measures at least " $\leq 5$ " $\mathrm{cm}$ in diameter at the femoral puncture site. Major bleeding was defined as a fall in hemoglobin of more than $3 \mathrm{gm} / \mathrm{dL}$ or any bleeding requiring a blood transfusion.

A technical success was when the invasive pulmonary pressure decreased by $25 \%$ and there was no clot in the main pulmonary branch.

Primary technical success was defined as the moment when CDT reached the end points, and secondary technical success was defined as the moment when CDT was followed by MT. 
Clinical success was an improvement in at least one clinical category of the New York Heart Association classification.

\section{Statistical analysis}

Statistical analysis was performed using commercially available Graph Pad Prism 8.0 software (USA). Each continuous variable was expressed as the mean \pm standard deviation or as the median (interquartile range), as appropriate. Each categorical variable was expressed as a percentage. The various patient cohorts were compared using either the Mann-Whitney test or the Kruskal-Wallis test. Probability values not exceeding 0.05 were considered significant.

\section{Results}

In total, 80 patients with submassive and massive pulmonary embolism were treated with CDT and MT. Demographic and clinical data and etiology of the pulmonary emboli are summarized in Table 1.

Angiographic and procedural results are summarized in Table 2.

In total, 80 patients with a mean age of $59 \pm$ \pm 16.8 years were treated. CDT was successful after the first post-operative day in $72(90 \%)$ patients, but thrombus aspiration and fragmentation was performed due to failed thrombolysis in $8(10 \%)$ patients. Final technical and clinical success was reached in $79(98.8 \%)$ and $77(96.3 \%)$ patients, respectively. The mean CDT time in IPE was $27.8 \pm 9.6 \mathrm{~h}$. The pulse rate and systolic blood pressure at presentation was $104.4 \pm 17.62 \mathrm{bpm}$ and $128.9 \pm 18.7 \mathrm{mmHg}$, respectively. Invasive pulmonary pressure dropped from $57.5 \pm 16.7$ to $39.9 \pm 13.3$ and finally to $38.9 \pm 13.5$ ( $p<0.001)$. Preand post-procedural pulmonary pressure is summarized in Figure 3. A caval filter was implanted in $4(5 \%)$ patients. Two patients received it in the acute phase $(2.5 \%)$ : one due to large iliac vein thrombosis and the other one due to recurrent $\mathrm{PE}$ with decreased pulmonary reserve. During follow up caval filter was implanted also in $2(2.5 \%)$ patients. The indication was in both patient's contraindication of oral anticoagulation due to major bleeding under post-procedural oral anticoagulation therapy (one gynecological and one cerebral focus).

Echocardiographic parameters are summarized in Table 3.

All patients had pre-procedural elevated right ventricular systolic pressure (55 [46-66] $\mathrm{mmHg}$ ), but due to left ventricular enlargement the typical D sign was not present in $4(5.0 \%)$ patients.
Table 1. Demographic data.

\begin{tabular}{lc}
\hline Age [years] & $59.0 \pm 16.8$ \\
Female & $38(47.5 \%)$ \\
Hypertension & $50(62.5 \%)$ \\
Hyperlipidemia & $39(48.8 \%)$ \\
Diabetes mellitus & $7(8.8 \%)$ \\
Obesity & $16(20.0 \%)$ \\
Active smokers & $4(5.1 \%)$ \\
Coronary artery disease & $3(3.8 \%)$ \\
Peripheral artery disease & $4(5.0 \%)$ \\
Renal failure & $18(22.5 \%)$ \\
Positive family history (PE) & $2(2.5 \%)$ \\
Causes of the deep venous thrombosis and PE \\
Idiopathic & $49(59.8 \%)$ \\
Tumor & $13(16.3 \%)$ \\
Oral anticonceptions & $2(2.5 \%)$ \\
Trauma or Immobility & $8(10.0 \%)$ \\
Hypercoagulability & $9(11.3 \%)$ \\
\hline
\end{tabular}

PE — pulmonary embolism

Table 2. Angiographic and procedural data.

\begin{tabular}{|c|c|}
\hline \multicolumn{2}{|l|}{ Angiographic data } \\
\hline \multicolumn{2}{|l|}{ Thrombi location: } \\
\hline Proximal main branch and central & $40(50.0 \%)$ \\
\hline Distal main branch & $36(45.0 \%)$ \\
\hline Distal segment & $4(5.0 \%)$ \\
\hline \multicolumn{2}{|l|}{ Thrombi side: } \\
\hline Right & $14(17.5 \%)$ \\
\hline Left & $15(18.8 \%)$ \\
\hline Both & $51(63.8 \%)$ \\
\hline \multicolumn{2}{|l|}{ Procedural data } \\
\hline Contrast consumption [mL] & $64.2 \pm 26.3$ \\
\hline Fluoroscopy time [min] & $4.77 \pm 1.9$ \\
\hline X-ray dose [dyn] & $358.1 \pm 170.1$ \\
\hline Dose area product $\left[\mathrm{dyn} / \mathrm{cm}^{2}\right]$ & $2.7 \pm 1.6$ \\
\hline Average lysis time $[\mathrm{h}]$ & $27.8 \pm 9.6$ \\
\hline Technical success & $79(98.8 \%)$ \\
\hline \multicolumn{2}{|l|}{ Procedural technique: } \\
\hline Catheter directed thrombolysis & $80(100.0 \%)$ \\
\hline Additional thrombectomy & $12(15.0 \%)$ \\
\hline Prolonged thrombolysis (> $24 \mathrm{~h}$ ) & $16(20.0 \%)$ \\
\hline Balloon dilatation & $2(2.5 \%)$ \\
\hline \multicolumn{2}{|l|}{ Invasive pulmonary pressure [mmHg]: } \\
\hline Before thrombolysis & $57.5 \pm 16.7$ \\
\hline First postoperative day & $39.9 \pm 39.9^{*}$ \\
\hline $\begin{array}{l}\text { Second postoperative day } \\
\text { or FINAL pressure }\end{array}$ & $38.9 \pm 13.5^{*}$ \\
\hline
\end{tabular}

${ }^{*} p<0.01$ 


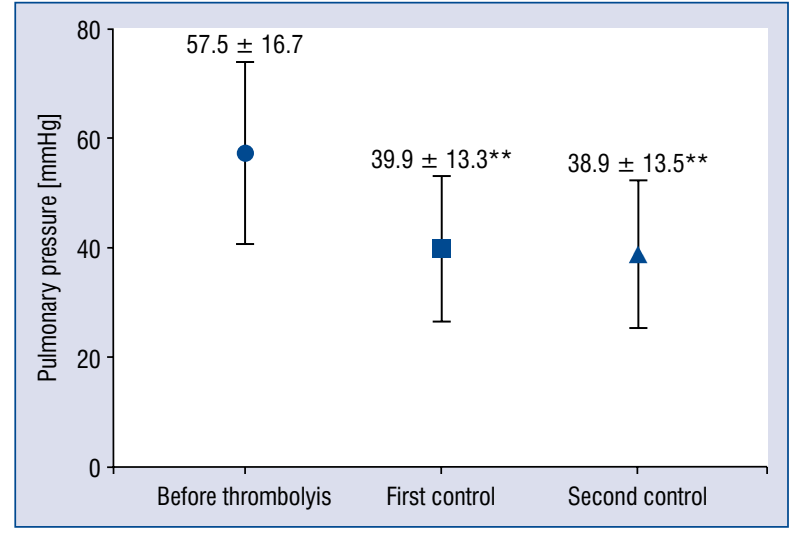

Figure 3. Invasive systolic pulmonary pressures before and after thrombolysis; ${ }^{*} \mathrm{p}<0.01$.

D sign was post-procedural not present in $93.8 \%$ and during follow-up in $95.0 \%$ of the patients. Tricuspid annular plane systolic excursion and pulmonary pressure has been improved after CDT and MT from 18 [16-20] to 22 [20-24] $\mathrm{mm}(\mathrm{p}=$ $=0.005)$ and from 55 [46-66] to $40[40-48] \mathrm{mmHg}$ $(\mathrm{p}<0.001)$, respectively.

Table 4.

Procedural complications are summarized in

As indicated in Table 3, intraprocedural complications were not observed. Worsening heart failure or renal failure was not detected in the investigated population. The rates of major and minor vascular access complications were $7.5 \%$ (4 major subcutaneous hematomas and 2 retroperitoneal hematomas) and 7.5\% (6 subcutaneous hematomas), respectively.

\section{One-year follow-up}

Clinical success at discharge was reached in $77(96.2 \%)$ patients. The 1-year major adverse events and cardiovascular mortality rates were $4 \%$ and $1.4 \%$, respectively. The overall 1 -year mortality rate was $6.7 \%$ (1 cardiovascular and 5 tumor-associated).

\section{Discussion}

According to available research, this study is the first to show long-term clinical benefit in patients who underwent CDT and MT in IPE through long-term analysis.

Systemic full dose thrombolysis in a recent meta-analysis reduced the risk for death and cardiovascular collapse but increased the risk for major bleeding and hemorrhagic stroke [4]. The PEITHO study was a randomized, double blind study of 1,005 submassive PE patients comparing the thrombolytic agent tenecteplase with heparin to a placebo with heparin [5]. Death or hemodynamic decompensation occurred within 7 days from randomization in $2.6 \%$ of the patients in the tenecteplase group compared to $5.6 \%$ in the placebo group. However, stroke occurred in $2.4 \%$ in the tenecteplase group compared to $0.2 \%$ in the placebo group. The long-term follow-up in the PEITHO study showed no survival (98.3\% vs. $98 \%)$ or functional (36\% vs. $20.1 \%$ ) or echocardiographic benefit in the thrombolysis arm compared with the anticoagulation arm [6].

Catheter interventions using thrombolytic drugs include CDT and pharmacomechanical thrombolysis. Their application was introduced due to a high rate of bleeding events during sys-

Table 3. Echocardiographic parameters.

\begin{tabular}{lcccc}
\hline ECHO & $\begin{array}{c}\text { Preinterventional } \\
(\mathbf{n}=\mathbf{8 0})\end{array}$ & $\begin{array}{c}\text { Post interventional } \\
(\mathbf{n}=\mathbf{8 0})\end{array}$ & $\begin{array}{c}\text { One-year follow-up } \\
(\mathbf{n}=\mathbf{7 4 )}\end{array}$ & $\mathbf{P}$ \\
\hline LVEF [\%] & $57[53-60]$ & $58[54-60]$ & $60[56-60]$ & $<0.001$ \\
PP & $55[46-66]$ & $40[40-48]$ & $35[30-40]$ & $<0.001$ \\
D sign: & & & & $<0.001$ \\
$\quad 0$ & $4(5.0 \%)$ & $75(93.8 \%)$ & $76(95.0 \%)$ & \\
1 & $76(95.0 \%)$ & $5(6.2 \%)$ & $4(5.0 \%)$ & \\
RV/LV: & & & & \\
$<1$ & $4(5.0 \%)$ & $51(63.8 \%)$ & $71(95.9 \%)$ & \\
$\cong 1$ & $21(26.3 \%)$ & $24(30.0 \%)$ & $0(0.0 \%)$ & \\
$>1$ & $55(68.8 \%)$ & $5(6.3 \%)$ & $3(4.1 \%)$ & \\
TAPSE & $18[16-20]$ & $22[20-24]$ & $24[22-27]$ & 0.005 \\
\hline
\end{tabular}

LV — left ventricle; LVEF — left ventricular ejection fraction; RV — right ventricle; PP — pulmonary pressure; TAPSE — tricuspid annular plane systolic excursion 
Table 4. Procedural complications and major adverse events (MAE).

\begin{tabular}{lcc}
\hline & One-month follow-up & One-year follow-up \\
\hline Summary of MAE & $0(0 \%)$ & $1(1.4 \%)$ \\
Cardiovascular death & $1(1.2 \%)$ & $2(2.7 \%)$ \\
Intracranial bleeding & $0(0 \%)$ & $0(0 \%)$ \\
Myocardial infarction & $0(0.0 \%)$ & $1(1.3 \%)$ \\
Repeat revascularization & $1(1.2 \%)$ & $3(4.0 \%)$ \\
All MAE's & $1(1.2 \%)$ & $5(6.7 \%)$ \\
Death (all) & $0(0 \%)$ & - \\
Periprocedural complication & $0(0 \%)$ & - \\
Respiratory failure & $1(1.2 \%)$ & - \\
Cardiogenic shock & & - \\
Stroke/Cerebral bleeding & $0(0 \%)$ & - \\
Pulmonary vascular complications & $0(0 \%)$ & \\
Perforation & & $2(2.7 \%)$ \\
Major distal embolization & $1(1.2 \%)$ & $6(7.5 \%)$ \\
Bleeding complications & & $6(7.5 \%)$ \\
Intracranial bleeding & $6(7.5 \%)$ & $13(16.2 \%)$ \\
Access site complications: & $6(7.5 \%)$ & \\
$\quad$ Major & $13(16.2 \%)$ & \\
$\quad$ Minor & & \\
All bleeding complications &
\end{tabular}

temic thrombolysis. The principal mechanism of transcatheter therapy is to either dissolve and/or directly remove the clot with direct thrombus aspiration, fragmentation, administration of local thrombolytic drug or combination of these mechanisms. Thrombus removal is applied to immediately restore blood flow to pulmonary circulation, with subsequent improvement in the right ventricular strain, hemodynamic status, and oxygenation. Today, the choice lies between streptokinase, recombinant tissue plasminogen activator and urokinase. The choice of procedure and tools differs based on the severity of the PE and estimated bleeding risk [7]. There are only a few prospective clinical trials examining CDT alone [8-10], ultrasound facilitated CDT [11, 12] or CBT with thrombus fragmentation and/or mechanical thrombectomy $[12,13]$ in the setting of intermediate-risk PE, but a head to head comparison of different techniques remains non-existant.

The main advantages of CDT are simplicity, high effectiveness and relatively low bleeding risk. The goal for CDT is to achieve similar efficacy to systemic thrombolysis and potentially decrease the rate of major and intracranial bleeding by delivering a significantly lower dose of thrombolytic drug directly into the thrombus over an extended period of time (12-24 h) through a pig-tail or dedicated thrombolytic catheter. The MAPPET-3 trial was the first CDT controlled trial in submassive PE using $100 \mathrm{mg}$ alteplase plus heparin or heparin only. The incidence of in-hospital mortality was significantly higher in the heparin-plus-placebo group than in the heparin plus alteplase group $(p=0.006)$, and the probability of 30-day event-free survival was higher in the heparin-plus-alteplase group $(\mathrm{p}=0.005)$ [8]. The MOPETT study was a randomized controlled trial of 121 patients comparing 'safe dose' thrombolytic (half of the full dose) with anticoagulation and an anticoagulation only group. Pulmonary artery pressures were significantly lower after $48 \mathrm{~h}$ in the thrombolytic group than in the anticoagulation group (16 vs. $10 \mathrm{mmHg}$ reduction, $\mathrm{p}<0.001$ ) [9]. In a recent publication by Carrmona et al. [10], local low-dose urokinase thrombolysis was done in submassive $\mathrm{PE}$ and an initially high pulmonary artery pressure after CTD (systolic 52.4 vs. 35.2 [17.2, 95\% CI 14.5-19.9]), and follow-up ultrasound showed improvement in right ventricular dysfunction. No life-threatening hemorrhagic complications were reported. The 6-month survival was $96.5 \%$ [10]. 
The present study shows similar results, but the primary end-point was reached only in $90 \%$ of patients with CDT, and additional thombectomy was done in $9.6 \%$ of the patients to achieve the primary end-point, which was reached finally in $100 \%$ of the patients.

The main disadvantages of CDT are still the high bleeding risk [7] and the limited effectiveness of the thrombolytic drug in old thrombi. Bleeding can be puncture related or due to the systemic effect of the thrombolytic drug. Puncture related complications can be reduced by ultrasound assisted femoral vein puncture [14]; however, in the current study population, traditional femoral vein puncture was used, and the rate of access site bleeding was relatively high. Periprocedural major bleeding (intracerebral or gastrointestinal) was not observed; however, 2 patients had major cerebral bleeding at the 1-year follow-up after initiation of novel anticoagulants. The results of a recent metaanalysis of 35 interventional and observational ("real world") studies on CDT calculated a pooled rate of $6.6 \%$ for major bleeding, with a $3.9 \%$ major bleeding and $2.6 \%$ in hospital mortality rate in the "stable hemodynamic status" group [15].

Ultrasound accelerated thrombolysis can be more effective than simple local thrombolysis and can reduce the bleeding complications, but a head to head comparison has not been done comparing the two techniques. The phase 2 ULTIMA (Ultrasound Accelerated Thrombolysis of Pulmonary Embolism) trial randomized 59 patients with acute main- or lower-lobe PE and an echocardiographic right-to-left ventricular dimension ratio of 1.0 to receive unfractionated heparin plus a catheter-directed, ultrasound-assisted thrombolytic regimen of 10 to $20 \mathrm{mg}$ recombinant tissue plasminogen activator over $15 \mathrm{~h}$, as opposed to heparin alone. Reduced-dose local thrombolysis significantly reduced the subannular right-to-left ventricular dimension ratio between the baseline and a 24-h follow-up without an increase in bleeding complications [11]. The PERFECT registry, which also lacked a control arm, included 101 patients with high-risk and intermediate-risk PE. There were no major or intracranial bleeding events with CDT [16].

Aspiration thrombectomy is a new treatment method and is the rational choice to aspirate thrombi from the vascular bed without using a thrombolytic. The potential complication is the distal embolization and pulmonary artery trauma, and the effectiveness is not well known. MT can be done with dedicated devices or with a simple guiding catheter [12]. In the present study group, aspiration thrombectomy was applied in 12 patients with a $100 \%$ technical and $90 \%$ clinical success without escalation of major bleeding events.

\section{Limitations of the study}

The main limitation of the study is the lack of a randomized control arm. Another limitation is that right heart catheterization was not performed in the patient after the 1-year follow-up.

\section{Conclusions}

Catheter directed thrombolysis in submassive PE had excellent results. However, additional mechanical thrombectomy and angioplasty was necessary in several patients to achieve a good clinical outcome.

\section{Conflict of interest: None declared}

\section{References}

1. Konstantinides SV, Torbicki A, Agnelli G, et al. Task Force for the Diagnosis and Management of Acute Pulmonary Embolism of the European Society of Cardiology (ESC). 2014 ESC guidelines on the diagnosis and management of acute pulmonary embolism. Eur Heart J. 2014; 35(43): 3033-69, 3069a, doi: 10.1093/eurheartj/ehu283, indexed in Pubmed: 25173341.

2. Jaff MR, McMurtry MS, Archer SL, et al. American Heart Association Council on Cardiopulmonary, Critical Care, Perioperative and Resuscitation, American Heart Association Council on Peripheral Vascular Disease, American Heart Association Council on Arteriosclerosis, Thrombosis and Vascular Biology. Management of massive and submassive pulmonary embolism, iliofemoral deep vein thrombosis, and chronic thromboembolic pulmonary hypertension: a scientific statement from the American Heart Association. Circulation. 2011; 123(16): 1788-1830, doi: 10.1161/CIR.0b013e318214914f, indexed in Pubmed: 21422387.

3. Hoeper MM, Bogaard HJ, Condliffe R, et al. Definitions and diagnosis of pulmonary hypertension. J Am Coll Cardiol. 2013; 62(25 Suppl): D42-D50.

4. Marti C, John G, Konstantinides S, et al. Systemic thrombolytic therapy for acute pulmonary embolism: a systematic review and meta-analysis. Eur Heart J. 2015; 36(10): 605-614, doi: 10.1093/ eurheartj/ehu218, indexed in Pubmed: 24917641.

5. Meyer G, Vicaut E, Danays T, et al. Fibrinolysis for Patients with Intermediate-Risk Pulmonary Embolism. N Engl J Med. 2014; 370(15): 1402-1411, doi: 10.1056/nejmoa1302097.

6. Konstantinides SV, Vicaut E, Danays T, et al. Impact of thrombolytic therapy on the Long-Term outcome of intermediaterisk pulmonary embolism. J Am Coll Cardiol. 2017; 69(12): 1536-1544, doi: 10.1016/j.jacc.2016.12.039, indexed in Pubmed: 28335835.

7. Abraham P, Arroyo DA, Giraud R, et al. Understanding haemorrhagic risk following thrombolytic therapy in patients with intermediate-risk and high-risk pulmonary embolism: a hy- 
pothesis paper. Open Heart. 2018; 5(1): e000735, doi: 10.1136/ openhrt-2017-000735, indexed in Pubmed: 29531763.

8. Konstantinides S, Geibel A, Heusel G, et al. Heparin plus alteplase compared with heparin alone in patients with submassive pulmonary embolism. N Engl J Med. 2002; 347(15): 1143-1150, doi: 10.1056/NEJMoa021274, indexed in Pubmed: 12374874 .

9. Sharifi M, Bay C, Skrocki L, et al. Moderate pulmonary embolism treated with thrombolysis (from the "MOPETT" Trial). Am J Cardiol. 2013; 111(2): 273-277, doi: 10.1016/j.amjcard.2012.09.027, indexed in Pubmed: 23102885.

10. Carmona SA, Redondo MP, Franco LN, et al. Local low-dose urokinase thrombolysis for the management of haemodynamically stable pulmonary embolism with right ventricular dysfunction. EuroIntervention. 2018; 14(2): 238-246, doi: 10.4244/ eij-d-17-00544.

11. Kucher N, Boekstegers P, Müller OJ, et al. Randomized, controlled trial of ultrasound-assisted catheter-directed thrombolysis for acute intermediate-risk pulmonary embolism. Circulation. 2014; 129(4): 479-486, doi: 10.1161/CIRCULATIONAHA.113.005544, indexed in Pubmed: 24226805.

12. Piazza G, Hohlfelder B, Jaff MR, et al. A prospective, singlearm, multicenter trial of ultrasound-facilitated, catheter-directed, low-dose fibrinolysis for acute massive and submassive pulmonary embolism: the SEATTLE II study. JACC Cardiovasc Interv. 2015; 8(10): 1382-1392, doi: 10.1016/j.jcin.2015.04.020, indexed in Pubmed: 26315743.

13. Ciampi-Dopazo JJ, Romeu-Prieto JM, Sánchez-Casado M, et al. Aspiration thrombectomy for treatment of acute massive and submassive pulmonary embolism: initial single-center prospective experience. J Vasc Int Radiol. 2018; 29(1): 101-106.

14. Yamagata K, Wichterle D, Roubícek T, et al. Ultrasound-guided versus conventional femoral venipuncture for catheter ablation of atrial fibrillation: a multicentre randomized efficacy and safety trial (ULTRA-FAST trial). Europace. 2018; 20(7): 1107-1114, doi: 10.1093/europace/eux175, indexed in Pubmed: 28575490.

15. Bajaj NS, Kalra R, Arora P, et al. Catheter-directed treatment for acute pulmonary embolism: Systematic review and single-arm meta-analyses. Int J Cardiol. 2016; 225: 128-139, doi: 10.1016/j. ijcard.2016.09.036, indexed in Pubmed: 27718446.

16. Kuo WT, Banerjee A, Kim PS, et al. Pulmonary embolism response to fragmentation, embolectomy, and catheter thrombolysis (PERFECT): initial results from a prospective multicenter registry. Chest. 2015; 148(3): 667-673, doi: 10.1378/ chest.15-0119, indexed in Pubmed: 25856269. 